\title{
Hepatoprotective Constituents in Plants 14. Effects of Soyasapogenol B, Sophoradiol, and Their Glucuronides on the Cytotoxicity of tert-Butyl Hydroperoxide to HepG2 Cells ${ }^{1)}$
}

\author{
Junei KinJo, ${ }^{*}, a$ Tomoki Hirakawa, ${ }^{b}$ Ryota Tsuchihashi, ${ }^{b}$ Tsuneatsu NagaO, ${ }^{a}$ Masafumi Okawa, ${ }^{b}$ \\ Toshihiro NoHARA, ${ }^{b}$ and Hikaru OKABE ${ }^{a}$ \\ ${ }^{a}$ Faculty of Pharmaceutical Sciences, Fukuoka University; 8-19-1 Nanakuma, Jonan-ku, Fukuoka 814-0180, Japan: and \\ ${ }^{b}$ Faculty of Pharmaceutical Sciences, Kumamoto University; 5-1 Oe-honmachi, Kumamoto 862-0973, Japan. \\ Received April 4, 2003; accepted May 16, 2003
}

\begin{abstract}
The effects of soyasapogenol B, sophoradiol, their glucuronides, and glycyrrhizin on the hepatotoxicity of tert-butyl hydroperoxide $(t$-BuOOH) in a human-liver-derived cell line (HepG2 cells) were investigated. Glycyrrhizin showed significant dose-dependent protective effects against the cytotoxicity of $t$-BuOOH. Among soyasapogenol $B$ and its glucuronides, the monoglucuronide showed the most potent hepatoprotective activity, followed by soyasapogenol B itself. Soyasaponin III was weakly protective, while soyasaponin I increased the toxicity of $t$-BuOOH. Among sophoradiol and its glucuronides, sophoradiol itself showed the most potent hepatoprotective activity, which was equal to glycyrrhizin, while the monoglucuronide and kaikasaponin III showed an increase in cytotoxicity. These results were considerably different from those reported previously on the protective effects of these compounds using primary cultures of immunologically injured rat liver cells. Consequently, the hepatoprotective action of the triterpene derivatives investigated would be different in HepG2 cells and in rat primary hepatocyte cultures.
\end{abstract}

Key words hepatoprotective triterpenoid; HepG2 cell; tert-butyl hydroperoxide; soyasapogenol B glucuronide; sophoradiol glucuronide

Saponins are glycosidic compounds present in many edible and inedible plants. Structurally, they are composed of a lipid-soluble aglycone consisting of either a sterol or, more commonly, a triterpenoid and water-soluble sugar residues differing in type and amount of sugars. ${ }^{2)}$ Because of their amphiphilic nature, they are highly surface-active. Their biological activity is closely related to the chemical structures that determine the polarity, hydrophobicity, and acidity of compounds. ${ }^{2)}$ A recent review by Safayhi and Sailer shows that pentacyclic triterpenes, including the oleanane skeleton, might be a rich natural resource of lead compounds for antiinflammatory drug development. ${ }^{3)}$ Some oleanane-type triterpenoidal saponins are known to exhibit hepatoprotective actions. ${ }^{3)}$ Saikosaponins ${ }^{4-6)}$ and glycyrrhizin ${ }^{7-9)}$ are the most well-known of these. In Japan, glycyrrhizin has been widely used as a drug to treat hepatitis. ${ }^{3)}$ Oleanene glucuronide (OG) is a triterpenoidal saponin of the olean-12-ene type, with a methyl group at $\mathrm{C}-17$ and a glucuronic acid moiety at the C-3 hydroxy group of the triterpene. ${ }^{10)}$ Although glycyrrhizin belongs to $\mathrm{OG}$, soyasaponin $\mathrm{I}^{11)}$ is a representative OG.

Puerariae Flos, the flowers of Pueraria lobata, is a crude drug used to counteract the overconsumption of alcohol in traditional Japanese and Chinese therapeutic systems. ${ }^{12)} \mathrm{We}$ have found that the total saponin fraction in this crude drug was effective in an experimental in vivo model of hepatic injury. ${ }^{13)}$ Abri Herba, the whole plant of Abrus cantoniensis, is used as a folk medicine for hepatitis in China. Its efficacy has been substantiated clinically. ${ }^{14)}$ We reported that the total saponin fraction and soyasaponin I isolated from this plant were effective for experimental liver injuries in vivo induced by $\mathrm{CCl}_{4}{ }^{15)}$ We have also reported the hepatoprotective activity of soyasaponin I and kaikasaponin III isolated from this drug against $\mathrm{CCl}_{4}$-induced cytotoxicity in primary cultured rat hepatocytes. ${ }^{16)}$

In a series of studies of hepatoprotective drugs, we devised an in vitro assay method in which the extent of immunological liver injury was measured in rat primary hepatocytes cultures. ${ }^{17)}$ We examined the preventive effect of soyasapogenol $\mathrm{B}^{18,19)}$ and sophoradiol ${ }^{1)}$ glucuronides using this method, and elucidated some structure-activity relationships.

However, because of the expense and the time involved, the use of primary cultured rat hepatocytes is not convenient for the screening of a large number of plant extracts and constituents. Therefore, Thabrew et al. have devised a reproducible microplate screening assay method, based on the quantitative measurement of cytotoxicity of tert-butyl hydroperoxide $(t$ - $\mathrm{BuOOH})$ against human-liver-derived HepG2 cells. ${ }^{20)}$ HepG2 cells have been suggested to be a useful in vitro model for investigation of the metabolism and toxicity of drugs compared with animal cells, since HepG2 cells retain many of the specialized functions which are characteristic of normal human hepatocytes, including the synthesis and secretion of plasma proteins. ${ }^{21)}$ In order to clarify the efficacy of soyasapogenol B and sophoradiol glucuronides and their aglycones on human-liver-derived cell lines, we investigated their hepatoprotective effects against the cytotoxicity of $t$ $\mathrm{BuOOH}$ in HepG2 cells.

\section{MATERIALS AND METHODS}

Instruments The instruments used in this study were the same as those described in previous papers. ${ }^{1,22)}$

Materials Soyasapogenol B and sophoradiol and their glucuronides have been isolated from some fabaceous plants according to previous papers. ${ }^{119)}$ Glycyrrhizin was purchased from Tokyo Chemical Ind. Co. (Tokyo, Japan). RPMI 1640 medium, fetal bovine serum (FBS), trypsin/EDTA, Dulbec- 
co's phosphate-bufferd saline (PBS), and kanamycin sulfate were purchased from Lifetech (Rockville, MD, U.S.A.). 70\% tert-Butyl hydroperoxide $(t-\mathrm{BuOOH})$ solution was obtained from Wako Chemical Co. (Osaka, Japan). AlamarBlue ${ }^{23)}$ was purchased from Nacalai Tesque (Kyoto, Japan).

Cells HepG2 cells were supplied from the Cell Resource Center for the Biomedical Research Institute of Development, Aging and Cancer, Tohoku University.

Determination of Hepatoprotective Activity The hepatoprotective activity was examined according to the method described by Thabrew. ${ }^{20)}$ HepG2 cells were cultured in $150 \mathrm{~cm}^{3}$ flasks at $37^{\circ} \mathrm{C}$ in a humidified atmosphere of $5 \%$ $\mathrm{CO}_{2} / 95 \%$ air. RPMI 1640 medium supplemented with $10 \%$ (v/v) FBS and kanamycin sulfate $(100 \mu \mathrm{g} / \mathrm{ml})$ was used for cultivation. Cells were routinely grown in monolayer until $80 \%$ confluence. Cell suspensions were prepared by rinsing a confluent monolayer with PBS and treating the cell layer with trypsin/EDTA for $4 \mathrm{~min}$. After inactivation of trypsin by FBS, the cells were centrifuged at $150 \times \mathbf{g}$ for $5 \mathrm{~min}$ and resuspended in the above medium. The cell suspension $(100 \mu \mathrm{l}$ per well) was placed in 96 -well plates $\left(3 \times 10^{4}\right.$ cells per well), and incubated at $37^{\circ} \mathrm{C}$ for $24 \mathrm{~h}$. After cultivation, dimethyl sulfoxide (DMSO) solution at various concentrations of the test samples was added to the wells. The final concentration of DMSO in the solution in each well was $1.6 \%$. The treatment with DMSO only was always used as a control (see below). After incubation for $1 \mathrm{~h}$, the $t$-BuOOH solution $(10 \mu \mathrm{l})$ was added to the wells. The $t$-BuOOH solution was adjusted to $1.25 \mathrm{~mm}$ in ice-cold RPMI 1640 medium immediately prior to addition. After $2 \mathrm{~h}$ incubation under the abovementioned conditions, cell viability was determined using AlamarBlue as described by Lancaster and Fields. ${ }^{24)}$ Cells in the plates were incubated at $37^{\circ} \mathrm{C}$ for $3 \mathrm{~h}$ after addition of AlamarBlue $(12 \mu \mathrm{l})$. Cell viability was determined by measuring the absorption of the reduced form of AlamarBlue using a 96-well microtiter plate reader at $570 \mathrm{~nm}(595 \mathrm{~nm}$ reference filter). Results are expressed as percentage protection, i.e., the percentage increase in cell viability compared to the viability of cells treated with the $t$ - $\mathrm{BuOOH}$ alone. The percent protection is calculated as $\{$ (Sample-Control)/(Reference-Control) $\} \times 100$. Reference is the absorbance value of those not challenged with $t-\mathrm{BuOOH}$ and samples. Control is the absorbance value of those challenged with $t$ - $\mathrm{BuOOH}$ and not treated with samples. The viability of HepG2 cells treated with $t$-BuOOH (1.25 mM) for $2 \mathrm{~h}$ was $30.1 \pm 0.8 \%$.

Statistical Analysis The data are shown as the mean \pm S.D. of four wells. After analysis of variances, Dunnett's test was employed to determine the significance of differences between control and experimental samples.

\section{RESULTS}

We examined the hepatoprotective activity of glycyrrhizin at first, since it has been successfully used to treat chronic hepatitis, ${ }^{3)}$ and it was proved to be hepatoprotective against in vitro immunological injury by antiserum in rat primary hepatocyte cultures. ${ }^{17)}$ Glycyrrhizin showed significant dose-dependent protective effects against the cytotoxicity of $t$ $\mathrm{BuOOH}$ in HepG2 cells (Fig. 1). For example, at the highest concentration $(500 \mu \mathrm{M})$, cell viability was improved significantly $(p<0.01)$ up to $52.2 \pm 5.7 \%$.

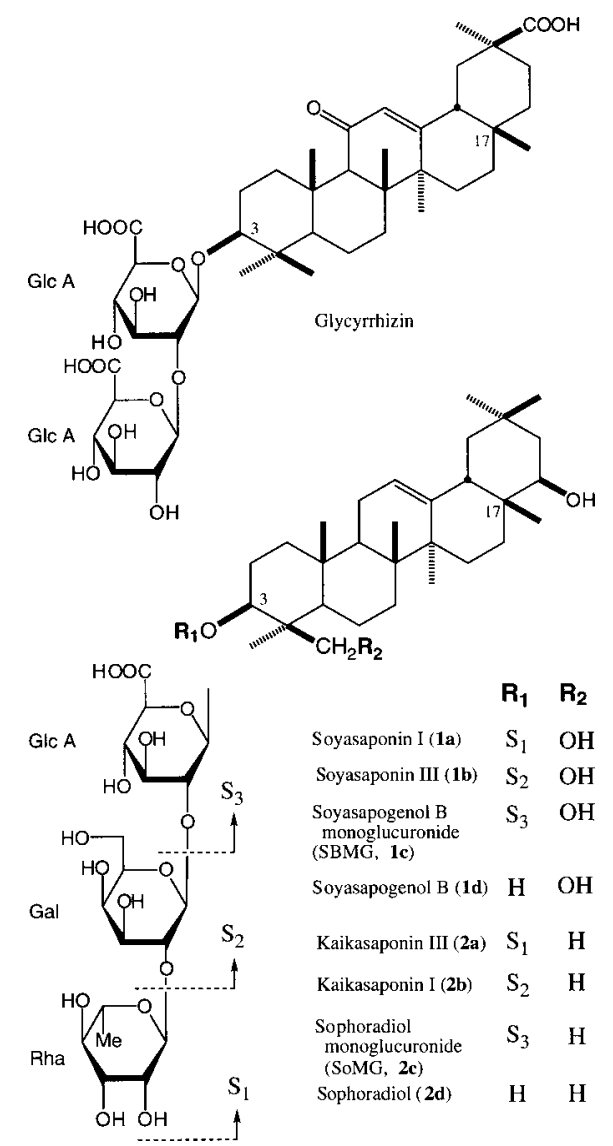

Next, we examined the hepatoprotective activity of several glucuronides which showed potent protective activity to in vitro immunological injury of rat primary hepatocyte cultures, ${ }^{1,19)}$ together with their aglycones, soyasapogenol B (1d) and sophoradiol (2d). The glucuronides investigated were soyasaponins I (1a), III (1b), soyasapogenol B monoglucuronide (SBMG, 1c), kaikasaponins III (2a), I (2b), and sophoradiol monoglucuronide (SoMG, 2c). The results are shown in Table 1.

Soyasaponin I (1a), a trisaccharide of 1d, did not show any hepatoprotective activity against the cytotoxicity of $t$ $\mathrm{BuOOH}$ in HepG2 cells, although it showed more potent hepatoprotective activity than glycyrrhizin in in vitro experiments using rat primary hepatocyte cultures and antiserum. ${ }^{17}$ In a similar manner, kaikasaponin III (2a), a trisaccharide of 2d and the most potent hepatoprotective trisaccharide in the rat model, also did not show any hepatoprotective activity against the cytotoxicity of $t-\mathrm{BuOOH}$ in HepG2 cells. In contrast, both trisaccharides showed strong hepatotoxicity (Table 1). The disaccharides [soyasaponin III (1b) and kaikasaponin I (2b)] of 1d and 2d exhibited greater effects than the trisaccharides in rat primary hepatocyte cultures. ${ }^{119)}$ However, $1 \mathbf{b}$ and $\mathbf{2 b}$ showed very weak protection of HepG2 cells against $t$-BuOOH. Similar to the experiment using rat primary hepatocyte cultures, the monoglucuronide (1c) of 1d showed moderate dose-dependent protective effects in HepG2 cells. On the contrary, the monoglucuronide (2c) of $\mathbf{2 d}$ demonstrated the strong hepatotoxicity at the higher dose in HepG2 cells, although it was slightly effective at $30 \mu \mathrm{M}$. On the other hand, soyasapogenol B (1d) itself showed weak protective activity at every dose, while sophoradiol (2d) exhibited signifi- 
Table 1. Hepatoprotective Activity of Glycyrrhizin, Compounds 1a-d and $\mathbf{2 a}-\mathbf{d}$

\begin{tabular}{|c|c|c|}
\hline Sample & Dose $(\mu \mathrm{M})$ & Protection (\%) \\
\hline \multirow[t]{5}{*}{ Glycyrrhizin } & 10 & $1.5 \pm 2.2$ \\
\hline & 30 & $6.9 \pm 2.0$ \\
\hline & 90 & $23.8 \pm 2.6 * *$ \\
\hline & 200 & $39.5 \pm 6.6^{* *}$ \\
\hline & 500 & $52.2 \pm 5.7 * *$ \\
\hline \multirow[t]{5}{*}{ Soyasaponin I (1a) } & 10 & $0.3 \pm 6.6$ \\
\hline & 30 & $0.3 \pm 5.1$ \\
\hline & 90 & $-12.4 \pm 4.2^{\dagger}$ \\
\hline & 200 & $-15.6 \pm 4.0^{\dagger}$ \\
\hline & 500 & $-32.8 \pm 3.5^{\dagger}$ \\
\hline \multirow[t]{5}{*}{ Soyasaponin III (1b) } & 10 & $-2.6 \pm 3.2$ \\
\hline & 30 & $1.9 \pm 7.0$ \\
\hline & 90 & $4.6 \pm 5.7$ \\
\hline & 200 & $12.6 \pm 7.0 *$ \\
\hline & 500 & $8.4 \pm 4.4$ \\
\hline \multirow{5}{*}{$\begin{array}{l}\text { Soyasapogenol B } \\
\text { monoglucuronide } \\
(\mathrm{SBMG}, \mathbf{1 c})\end{array}$} & 10 & $-1.5 \pm 4.0$ \\
\hline & 30 & $8.5 \pm 2.0$ \\
\hline & 90 & $22.9 \pm 8.8 * *$ \\
\hline & 200 & $28.5 \pm 4.9 * *$ \\
\hline & 500 & $32.4 \pm 10.1^{* *}$ \\
\hline \multirow[t]{5}{*}{ Soyasapogenol B (1d) } & 10 & $13.7 \pm 9.4 * *$ \\
\hline & 30 & $22.3 \pm 4.2 * *$ \\
\hline & 90 & $5.9 \pm 3.8$ \\
\hline & 200 & $21.3 \pm 4.1 * *$ \\
\hline & 500 & $15.9 \pm 2.4 * *$ \\
\hline \multirow[t]{5}{*}{ Kaikasaponin III (2a) } & 10 & $-1.1 \pm 4.6$ \\
\hline & 30 & $2.6 \pm 6.3$ \\
\hline & 90 & $-11.6 \pm 2.5^{\dagger}$ \\
\hline & 200 & $-22.1 \pm 5.1^{\dagger}$ \\
\hline & 500 & $-52.3 \pm 2.2^{\dagger}$ \\
\hline \multirow[t]{5}{*}{ Kaikasaponin I (2b) } & 10 & $1.1 \pm 4.3$ \\
\hline & 30 & $1.1 \pm 3.2$ \\
\hline & 90 & $0.0 \pm 3.4$ \\
\hline & 200 & $3.8 \pm 5.1$ \\
\hline & 500 & $8.4 \pm 4.9$ \\
\hline \multirow{5}{*}{$\begin{array}{l}\text { Sophoradiol } \\
\text { monoglucuronide } \\
(\text { SoMG, 2c) }\end{array}$} & 10 & $6.1 \pm 7.5$ \\
\hline & 30 & $16.6 \pm 6.7 * *$ \\
\hline & 90 & $-7.7 \pm 5.2$ \\
\hline & 200 & $-41.5 \pm 2.9^{\dagger}$ \\
\hline & 500 & $-54.2 \pm 1.1^{\dagger}$ \\
\hline \multirow{5}{*}{ Sophoradiol (2d) } & 10 & $8.8 \pm 4.5$ \\
\hline & 30 & $22.5 \pm 4.1 * *$ \\
\hline & 90 & $40.2 \pm 6.1^{* *}$ \\
\hline & 200 & $41.7 \pm 6.1 * *$ \\
\hline & 500 & $48.4 \pm 9.5^{* *}$ \\
\hline
\end{tabular}

Effect of samples on $t$-BuOOH (1.25 mm)-induced cytotoxicity in HepG2 cells. The percent protection is calculated as $\{($ Sample-Control $) /($ Reference-Control $)\} \times 100$. Reference is the absorbance value of those not challenged with $t-\mathrm{BuOOH}$ and samples. Control is the absorbance value of those challenged with $t$ - $\mathrm{BuOOH}$ and not treated with samples. Significantly different from control value, effective $* p<0.05, * * p<0.01$. Significantly different from control value, toxic ${ }^{\dagger} p<0.01$.

cant dose-dependent protective effects in HepG2 cells. The hepatoprotective activity of $\mathbf{2 d}$ at $500 \mu \mathrm{m}$ was equl to that of glycyrrhizin.

\section{DISCUSSION}

Among soyasapogenol B (1d) and its glucuronides (1ac), the decreasing order of the hepatoprotective effect on the cytotoxicity of $t$-BuOOH in HepG2 cells was monoglucuronide (1c) $>$ aglycone (1d) $>$ disaccharide (1b). The trisaccharide (1a) showed strong hepatotoxicity. In in vitro experiments using rat primary hepatocyte cultures and antiserum, the order of potency was $\mathbf{1 c} \geq \mathbf{1 b} \gg \mathbf{1 a} \geq \mathbf{1 d} .^{19)}$ The trisaccha- ride (1a) did not demonstrate any cytotoxicity in rat hepatocytes. ${ }^{19)}$ Among sophoradiol (2d) and its glucuronides (2ac), the aglycone (2d) itself showed the most potent hepatoprotective effect on human-liver-derived HepG2 cells that was equal to glycyrrhizin, which is used to treat chronic hepatitis. In the rat model, $2 \mathbf{d}$ showed very weak protective activity that was less than 1d. Although the trisaccharide (2a) did not show any cytotoxicity in rat hepatocytes, ${ }^{1)}$ it exhibited a strong hepatotoxicity in HepG2 cells similar to 1a. However, 1a and 2a did not exhibit hepatotoxicity in HepG2 cells without $t$-BuOOH (data not shown). Further, 1a and 2 a showed anticomplementary activity, i.e., inhibition of the hemolysis of human serum against sensitized erythrocytes. ${ }^{25}$ ) Consequently, the hepatoprotective action of the triterpenes investigated would be different in HepG2 cells and in rat primary hepatocyte cultures. The differense may be due the difference between human hepatocytes and rat hepatocytes.

Puerariae Flos ${ }^{13)}$ and Abri Herba ${ }^{14)}$ are known to be hepatoprotective crude drugs. Whereas three major saponins obtained from these crude drugs are weakly hepatoprotective kaikasaponin I (2b), or strongly hepatotoxic sophoradiol monoglucuronide (2c) and kaikasaponin III (2a). It seems that there might be some inconsistency in the facts. However, the hepatoprotective properties of these crude drugs could be reasonably attributed to sophoradiol (2d) generated from its glucuronides $(\mathbf{2} \mathbf{a}-\mathbf{c})$ by hydrolysis by intestinal bacteria. ${ }^{26)}$

Acknowledgements This work was supported by a Grant-in-Aid for Scientific Research (C) (No. 13672246) from the Ministry of Education, Culture, Sports, Science and Technology, Japan.

\section{REFERENCES AND NOTES}

1) Preceding paper: Kinjo J., Ikeda T., Okawa M., Udayama M., Hirakawa T., Shii Y., Nohara T., Biol. Pharm. Bull., 23, 1118-1121 (2000).

2) Rao A. V., Sung, M.-K., J. Nutrition, 125 (Suppl.), S717-724 (1995).

3) Safayhi H., Sailer E. R., Planta Medica, 63, 487-493 (1997).

4) Abe H., Sakaguchi M., Yamada M., Arichi S., Odashima S., Planta Medica, 40, 366-372 (1980).

5) Matsuda H., Murakami T., Ninomiya K., Inadzuki M., Yoshikawa M., Bioorg. Med. Chem. Lett., 7, $2193-2198$ (1997).

6) Yoshikawa M., Murakami T., Hirano K., Inadzuki M., Ninomiya K., Matsuda H., Tetrahedron Lett., 38, 7395-7398 (1997).

7) Kiso Y., Tohkin M., Hikino H., Hattori M., Sakamoto T., Namba T., Planta Medica, 50, 298-302 (1984).

8) Matsuda H., Samukawa K., Kubo M., Planta Medica, 57, 523-526 (1991).

9) Nose M., Ito M., Kamimura K., Shimizu M., Ogiwara Y., Planta Medica, 60, 136-139 (1994).

10) Kinjo J., Natural Medicines, 50, 79-85 (1996).

11) Kitagawa I., Yoshikawa M., Yosioka I., Chem. Pharm. Bull., 24, 121129 (1976).

12) Kinjo J., Takeshita T., Abe Y., Terada N., Yamashita H., Yamasaki M., Takeuchi K., Murakami K., Tomimatsu T., Nohara T., Chem. Pharm. Bull., 36, 1174-1179 (1988).

13) Niiho Y., Yamasaki T., Nakajima Y., Itoh H., Takeshita T., Kinjo J., Nohara T., Yakugaku Zasshi, 110, 604-611 (1990).

14) Chiang T. C., Chang H. M., Planta Medica, 46, 52-55 (1982).

15) Takeshita T., Ito Y., Sakai Y., Nohara T., Yasuhara M., Saito H., Kitagawa I., Ariga T., Irino N., Takaoka T., J. Pharmacobio-Dyn., 13, s-54 (1990).

16) Miyao H., Arao T., Udayama M., Kinjo J., Nohara T., Planta Medica, 64, 5-7 (1998).

17) Arao T., Udayama M., Kinjo J., Nohara T., Funakoshi T., Kojima S., Biol. Pharm. Bull., 20, 988 -991 (1997). 
18) Kinjo J., Imagire M., Arao T., Udayama M., Nohara, T., Planta Medica, 64, 233-236 (1998).

19) Ikeda T., Udayama M., Okawa M., Arao T., Kinjo J., Nohara T., Chem. Pharm. Bull., 46, 359-361 (1998).

20) Thabrew M. I., Hughes R. D., McFarlane I. G., J. Pharm. Pharmacol., 49, 1132-1135 (1997).

21) Sumida A., Yamamoto I., Zhou Q., Morisaki T., Azuma J., Biol. Pharm. Bull., 22, 61-65 (1999).

22) Kinjo J., Nagao T., Tanaka T., Nonaka G., Okabe, H., Biol. Pharm.
Bull., 24, 1443-1445 (2001).

23) Lancaster M. V., Fields R. D., U.S. Patent 5501959 (1996).

24) Lancaster M. V., Fields R. D., Am. Biotech. Laboratory, 11, 48-50 (1993).

25) Oh S.-R., Kinjo J., Shii Y., Ikeda T., Nohara T., Ahn K.-S., Kim J.-H., Lee H.-K., Planta Medica, 66, 506-510 (2000).

26) Hattori M., Sakamoto T., Kobashi K., Namba T., Planta Medica, 48, $38-42$ (1983). 